\title{
Die Leistungsfähigkeit bleibt erhalten
}

Fragestellung: Verbessert Propranolol die körperliche Belastbarkeit bei Patienten mit posturalem Tachykardiesyndrom?

Hintergrund: Betablocker werden bei einem posturalen Tachykardiesyndrom (POTS) häufig empfohlen, schränken als Nebenwirkung allerdings vielfach die körperliche Belastbarkeit ein. Der Effekt von $20 \mathrm{mg}$ Propranolol im Vergleich zu Placebo auf die maximale Sauerstoffaufnahme bei gesunden Probanden und Patienten mit POTS soll daher getestet werden.

Patienten und Methodik: Elf Patienten mit POTS und sieben gesunde Kontrollpersonen wurden randomisiert und erhielten doppelblind entweder $20 \mathrm{mg}$ Propranolol oder Placebo. Es folgte ein Belastungstest auf einem Fahrradergometer, um als primäre Outcome-Variable die maximale Sauerstoffaufnahme $\left(\mathrm{VO}_{2 \text { max }}\right)$ als Maß für die körperliche Belastbarkeit bestimmen zu können. In einem weiteren Protokoll wurde nach einer Auswaschphase ebenfalls der Einfluss von höher dosierten Propranolol ( $80 \mathrm{mg}$ ) oder Metoprolol (100 mg) im Vergleich zu Placebo beurteilt.

Ergebnisse: Die $\mathrm{VO}_{2 \max }$ war bei allen Placebogruppen nicht unterschiedlich. $20 \mathrm{mg}$ Propranolol verbesserte die $\mathrm{VO}_{2 \text { max }}$ in $\mathrm{Pa}$ tienten mit POTS, aber nicht bei gesunden Kontrollpersonen $(24,5 \pm 0,7$ vs. $27,6 \pm 1,0 \mathrm{ml} / \mathrm{min} / \mathrm{kg} ; \mathrm{p}=0,024)$. Der Anstieg der $\mathrm{VO}_{2 \text { max }}$ ging mit einer reduzierten Spitzenherzfrequenz (142 \pm 8 vs. $164 \pm 4 ; \mathrm{p}=0,005)$ und einem erhöhtem Schlagvolumen $(81 \pm 4$ vs. $67 \pm 3 \mathrm{ml} ; \mathrm{p}=0,013)$ in der Gruppe mit $20 \mathrm{mg}$ Propranolol im Vergleich zu Placebo einher. Weder $80 \mathrm{mg}$ Propranolol noch $100 \mathrm{mg}$ Metoprolol verbesserten die $\mathrm{VO}_{2 \text { max }}$, obwohl eine vergleichbare Herzfrequenzreduktion erreicht wurde.
Arnold AC, Okamoto LE, Diedrich $A$ et al. Low-dose propranolol and exercise capacity in postural tachycardia syndrome. A randomized study. Neurology 2013; 80: 1927-33
Schlussfolgerungen: Niedrig dosiertes Propranolol (20 $\mathrm{mg}$ ) reduziert bei Patienten mit POTS die Herzfrequenz ohne die maximale Sauerstoffaufnahme unter Belastung einzuschränken.

\section{-Kommentar von Klaus Gröschel, Mainz}

\section{Therapieversuch gerechtfertigt}

Bei dem posturalem Tachykardiesyndrom (POTS) handelt es sich um eine Erkrankung, die überwiegend weibliche Jugendliche oder Frauen bis zum Alter von 50 Jahren betrifft und durch eine ausgeprägte Tachykardie (> 30 Schläge/Minute Anstieg oder > 120 Schläge/Minute innerhalb von 10 Minuten) im Stehen gekennzeichnet ist. Dies kann zu einer orthostatischen Intoleranz führen, ohne zwingend in einer Synkope enden zu müssen. Neben dem unbedenklich zu empfehlenden körperlichem Ausdauertraining sorgt auch eine medikamentöse The- rapie mit unselektiven Betablockern für eine Herzfrequenzreduktion und somit einer besseren posturalen Toleranz. Leider schränken Betablocker als unerwünschte Nebenwirkung die sportliche Leistungsfähigkeit ein, sodass im Endeffekt eine Steigerung der Lebensqualität nicht generell gegeben ist. In der hier vorgestellten, randomisierten Studie konnte nun nachgewiesen werden, dass eine niedrige Dosierung von Propranolol $(20 \mathrm{mg}$ ) im Vergleich zu Placebo eine Herzfrequenzabsenkung möglich macht, ohne die maximale Sauerstoffaufnahme unter Belastung einzuschränken. Höhere Dosierungen von Betablockern (Propranolol 80 mg oder Metoprolol 100 $\mathrm{mg}$ ) zeigten diesen Effekt nicht, sodass hier eine störende Einschränkung der körperlichen Belastbarkeit denkbar ist. In länger angelegten prospektiven Studien wird sich zeigen, ob sich die hier kurzfristig nachgewiesenen Änderungen auch in einer besseren Lebensqualität der Patienten wiederfinden. Die vorliegende Studie legt zumindest einen Therapieversuch mit 20 mg Propranolol, zusätzlich zu einem Ausdauertraining bei $\mathrm{Pa}-$ tienten mit POTS nahe, ohne dass dieser einen negativen Einfluss auf die körperliche Leistungsfähigkeit haben dürfte.

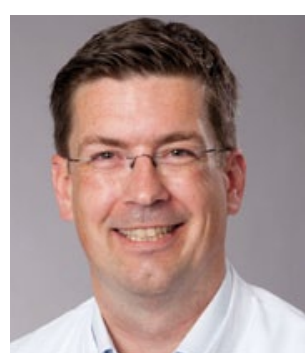

PD Dr. med. Klaus Gröschel, Mainz

Klinik und Poliklinik für Neurologie, Universitätsmedizin Mainz

E-Mail: klaus.groeschel@unimedizinmainz.de 